\title{
Use of Polymyxin B Hemoperfusion in a Patient with Septic Shock and Septic Car- diomyopathy Who Was Placed on Extracorporeal Membrane Oxygen Support
}

\author{
Sun Hye Shin, M.D. ${ }^{1}$, Hyun Lee, M.D. ${ }^{2}$, Aeng Ja Choi, A.P.N. ${ }^{3}$, Kylie Hae-Jin Chang, M.D. ${ }^{4}$, \\ Gee Young Suh, M.D., Ph.D. ${ }^{2,5}$, and Chi Ryang Chung, M.D. ${ }^{5}$ \\ ${ }^{1}$ Department of Medicine, ${ }^{2}$ Division of Pulmonary and Critical Care Medicine, ${ }^{3}$ Critical Care Nursing Team, ${ }^{4}$ Department of Obstetrics and Gynecology, \\ ${ }^{5}$ Department of Critical Care Medicine, Samsung Medical Center, Sungkyunkwan University School of Medicine, Seoul, Korea
}

\begin{abstract}
Although shock in sepsis is usually managed successfully by conventional medical treatment, a subset of cases do not respond and may require salvage therapies such as veno-arterial extracorporeal membrane oxygenation (VA ECMO) support as well as an attempt to remove endotoxins. However, there are limited reports of attempts to remove endotoxins in patients with septic shock on VA ECMO support. We recently experienced a case of septic shock with severe myocardial injury whose hemodynamic improvement was unsatisfactory despite extracorporeal membrane oxygenation (ECMO) support. Since the cause of sepsis was acute pyelonephritis and blood cultures grew gram-negative bacilli, we additionally applied polymyxin B direct hemoperfusion (PMX-DHP) to the ECMO circuit and were able to successfully taper off vasopressors and wean off ECMO support. To the best of our knowledge, this is the first adult case in which PMX-DHP in addition to ECMO support was successfully utilized in a patient with septic shock. This case indicates that additional PMX-DHP therapy may be beneficial and technically feasible in patients with septic shock with severe myocardial injury refractory to ECMO support.
\end{abstract}

Key Words: extracorporeal membrane oxygenation; hemoperfusion; polymyxin B; myocardia diseases; septic shock.

Sepsis is one of the leading causes of death in medical intensive care units, with increased mortality especially when accompanied by shock.[1] In most cases, shock in sepsis can be managed with conventional treatment, such as proper antibiotics and source control. Unfortunately, some patients do not respond and eventually require salvage therapies such as extracorporeal membrane oxygenation (ECMO) support[2-4] and, potentially, an attempt to remove endotoxins.[5]

Despite the controversy over the use of ECMO in adult patients with septic shock, recent studies show that ECMO may be effective in patients with refractory septic shock, particularly in the setting of severe myocardial injury.[2,3] An attempt to remove endotoxin using devices, such as polymyxin B-immobilized fiber cartridge, in septic shock patients is an established therapeutic option in Japan and some European countries.[5] The use of such devices has been associated with improved hemodynamics, blood oxygenation profile, organ function, and further on reduction in mortality.[6,7] Recently, a combination of ECMO support and blood purification therapy has been shown to remove pro-inflammatory cytokines, thereby preventing he-

Received on October 20, 2015 Revision Received on April 25, 2016 Accepted on May 4, 2016

Correspondence to: Chi Ryang Chung, Department of Critical Care Medicine, Samsung Medical Center, Sungkyunkwan University School of Medicine,

81 Irwon-ro, Gangnam-gu, Seoul 06351, Korea

TEL: +82-2-3410-3430, FAX: +82-2-2148-7088

Email: ccrzzang@gmail.com

*No potential conflict of interest relevant to this article was reported. modynamic deterioration and multi-organ failure in an animal model.[8] Moreover, a case of a patient with septic shock successfully managed with the combined use of a left ventricular assist device, ECMO, and CytoSorb hemofiltration device was reported[9] as well as a case of septic neonate under veno-arterial (VA) ECMO support rescued by polymyxin B direct hemoperfusion (Toraymyxin ${ }^{\circledR}$, Toray Industries, Tokyo, 
Japan).[10]

To the best of our knowledge, simultaneous PMX-DHP application in adult patients in septic shock under VA ECMO support has not been previously reported. Therefore, we report our experience of applying PMX-DHP to a patient in septic shock due to pyelonephritis with severe myocardial injury refractory to VA ECMO support.

\section{Case Report}

A 69-year-old female presented to the emergency department with fever, altered mental status, and right flank pain. She had been treated for hypertension and underwent surgery for spinal stenosis four years ago. Her initial vital signs were blood pressure $123 / 78 \mathrm{mmHg}$, heart rate (HR) 110 per minute (/min), respiration rate $24 / \mathrm{min}$ and body temperature $38.6^{\circ} \mathrm{C}$. Physical examination showed right costovertebral tenderness. The laboratory findings were significant for total leukocyte count of 14,370 cells/ $\mu \mathrm{L}$ (segmented neutrophil $79.5 \%$ ), platelet count of 163,000 cells $/ \mu \mathrm{L}$, total bilirubin of $1.9 \mathrm{mg} / \mathrm{dL}$, creatinine of $1.2 \mathrm{mg} / \mathrm{dL}, \mathrm{C}$-reactive protein of $44.29 \mathrm{mg} / \mathrm{dL}$, procalcitonin of $15.12 \mathrm{ng} / \mathrm{mL}$, lactate of $1.61 \mathrm{mmol} / \mathrm{L}$, troponin I of $1.872(\mathrm{ng} / \mathrm{mL})$, and pyuria on urine microscopy. Computed tomography scan revealed a ureteral stone with hydronephrosis in the right kidney (Fig. 1). Intravenous ceftriaxone was promptly initiated under the diagnosis of acute pyelonephritis and the patient also underwent emergent percutaneous nephrostomy (PCN).
However, she developed septic shock with type IV respiratory failure and acute kidney injury with metabolic acidosis. The patient was admitted to the intensive care unit for mechanical ventilation and continuous renal replacement therapy (CRRT). Volume resuscitation and administration of vasopressors and systemic steroids were performed, but shock did not respond to treatment. Bedside echocardiography indicated severely decreased left ventricular systolic function with global hypokinesia (left ventricular ejection fraction $<10 \%$ ). The mean arterial blood pressure (MAP) was $69 \mathrm{mmHg}(83 / 61 \mathrm{mmHg})$ and HR was 109/min under intravenous infusion of norepinephrine $0.5 \mathrm{mcg} / \mathrm{kg} / \mathrm{min}$ and vasopressin $0.03 \mathrm{IU} / \mathrm{min}$. After multidisciplinary discussion with the ECMO team, VA ECMO support was initiated. During the ECMO cannulation procedure, the patient suffered a cardiac arrest and cardiopulmonary resuscitation was performed for 2 minutes. Laboratory findings immediately following ECMO support revealed a lactate level of 22.2 $\mathrm{mmol} / \mathrm{L}$ and troponin I level of $36.5 \mathrm{ng} / \mathrm{mL}$. ECMO flow ranged from 4.0 to $5.0 \mathrm{~L} / \mathrm{min}$. However, high-dose vasopressor requirement and hypotension persisted even following 14 hours of ECMO support. The MAP was $65 \mathrm{mmHg}$ with no pulse pressure, HR was $79 / \mathrm{min}$. Follow-up laboratory tests revealed lactate level $14.2 \mathrm{mmol} / \mathrm{L}$ and troponin I level $42.1 \mathrm{ng} / \mathrm{mL}$. Initial blood and urine cultures reported gramnegative bacilli, which were later identified as Eschericha coli. Given that the source of sepsis was controlled with $\mathrm{PCN}$ and the causative organism was gram-negative, we connected the PMX-DHP, a hemofiltration device to the
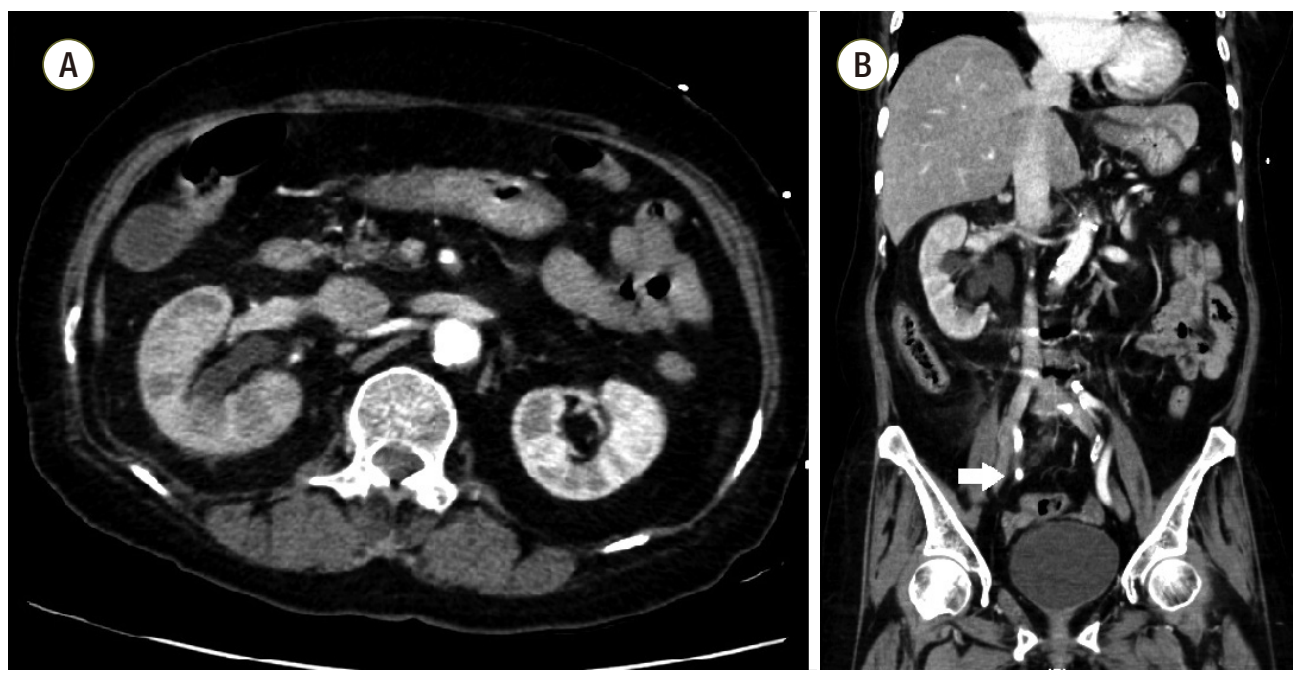

Fig. 1. Computed tomography of abdomen. (A) Multifocal perfusion decreased area and hydronephroureterosis of right kidney. (B) About 0.7 $\mathrm{cm}$ sized stone (white arrow) in right distal ureter which can be distinguished from calcification of adjacent vessels. 
CRRT device and to the VA ECMO circuit (Fig. 2) in hopes of removing any circulating endotoxin. The access lines of hemoperfusion and CRRT were connected to the arterial line of ECMO (Fig. 3A); the return lines of PMX-DHP and CRRT were connected to the venous line of ECMO (Fig. $3 \mathrm{~B})$. We set the ECMO flow to 4-5 L/min while PMX-DHP

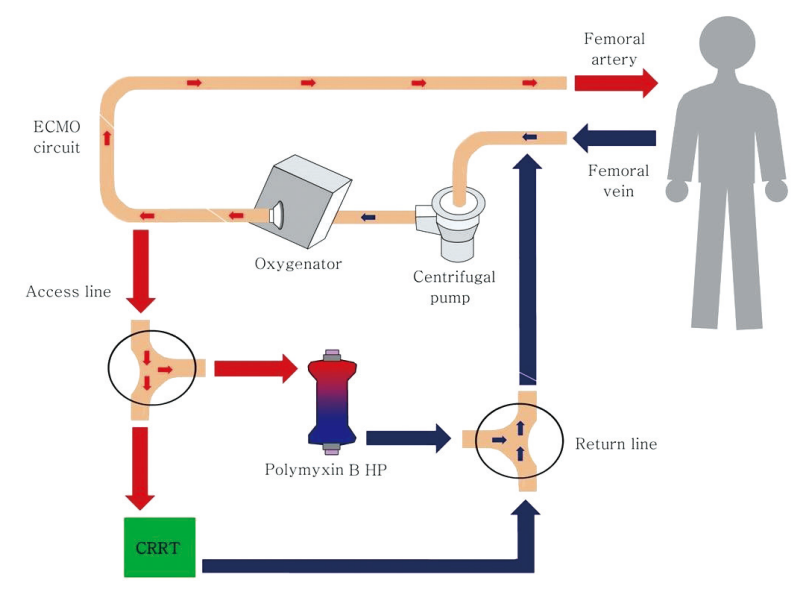

Fig. 2. Schematic diagram for simultaneous connection of ECMO, CRRT, and HP device. VA-ECMO was performed using the left femoral artery and right femoral vein. The access line and return line of CRRT and HP device were connected to the arterial line and venous line of ECMO circuit via three-way connector, respectively. ECMO: extracorporeal membrane oxygenation; CRRT: continuous renal replacement therapy; HP: hemoperfusion; VAECMO: veno-arterial extracorporeal membrane oxygenation. was applied. The flow of CRRT and hemofiltration ranged between $100 \mathrm{ml} / \mathrm{min}$ and $150 \mathrm{~mL} / \mathrm{min}$ with no significant adverse events or alarm signs indicating false connection between these devices. Neither membrane nor circuit clotting were observed, although anticoagulation was not performed because of progressive thrombocytopenia.

After 3 hours of the PMX-DHP session, we were able to reduce the norepinephrine dose down to half the initial dose and discontinue the vasopressin infusion. The MAP was 86 $\mathrm{mmHg}$ and a pulse pressure developed $(95 / 78 \mathrm{mmHg})$. Laboratory findings at 24 hours after PMX-DHP revealed significantly decreased lactate level to $9.1 \mathrm{mmol} / \mathrm{L}$ and troponin I level to $23.6 \mathrm{ng} / \mathrm{mL}$ (Fig. 4). On the fourth hospital day, improved left ventricular systolic function was documented on follow-up echocardiography and then VA-ECMO was disconnected successfully, only after 2 days and 15 hours of maintenance. Although she recovered from refractory septic shock, she suffered from vasopressor induced necrosis of the right hand, ECMO cannulation site dehiscence, and recurrent hematochezia due to ischemic and pseudomembraneous colitis. Unfortunately, she additionally experienced a non-ST segment elevated myocardial infarction on the twentieth hospital day and underwent coronary bypass graft surgery. Eventually, she was discharged to a local hospital for rehabilitation after a three month hospitalization (Fig. 5).
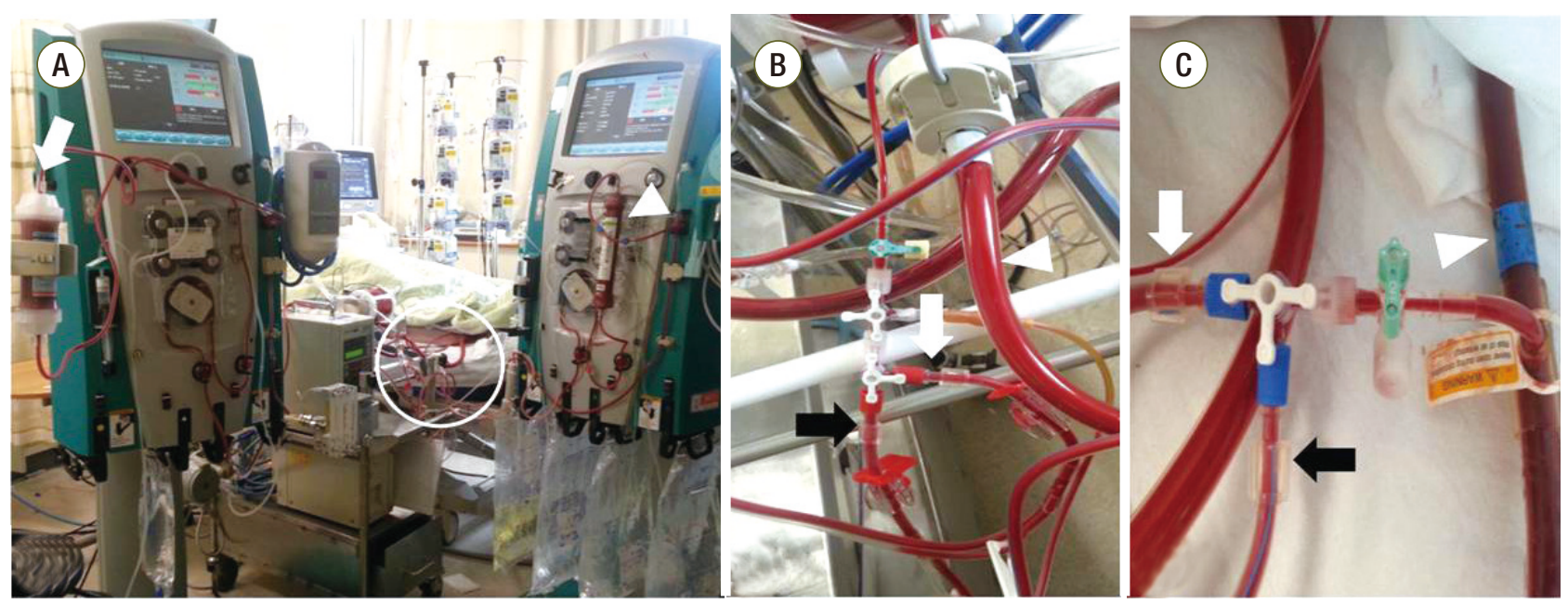

Fig. 3. Pictures showing the connection between devices. (A) A polymyxin B hemoperfusion device (white arrow) and CRRT (white arrow head) were connected to the ECMO circuit (white circle). (B) The access line of HP (black arrow) and CRRT (white arrow) were connected to the arterial line of ECMO (white arrow head). (C) The return line of HP (black arrow) and CRRT (white arrow) were connected to the venous line of ECMO (white arrow head). CRRT: continuous renal replacement therapy; ECMO: extracorporeal membrane oxygenation; HP: hemoperfusion. 


\section{Discussion}

There are two major hemodynamic disturbances associated with early death in septic shock.[11] One of them is distributive shock, which is characterized by low systemic vascular resistance with preserved cardiac index and the other is cardiogenic shock, which is characterized by decreased cardiac index.

The role of ECMO in adults with septic shock is still controversial.[2-4] It is well known that ECMO does not filter circulating inflammatory cytokines that can worsen septic shock,[12] and thus, ECMO appears to have little effect in patients with predominantly distributive shock, where heart function is preserved but the increased oxygen demand itself cannot be overcome by ECMO support.[3] Recent studies suggest that ECMO may be beneficial in patients with severe myocardial injury, as it may provide extra time to overcome the hemodynamic instability caused by myocardial dysfunction.[2,3] In the present case, the patient had severe myocardial injury with global hypokinesia and high troponin I levels, indicating that she may be a suitable candidate for ECMO support. Unfortunately, the hemodynamic support by ECMO was not enough to decrease the lactate level and vasopressor requirement. This may be due to inflammatory mediators causing ongoing distributive shock and septic cardiomyopathy.

During gram-negative bacterial infections, endotoxin (lipopolysaccharide) is recognized by toll-like receptor 4 , leading to the release of pro-inflammatory cytokines, which is followed by coagulopathy and vasodilation.[13,14] Polymyxin (PMX) is a cyclic cationic polypeptide antibiotic derived from Bacillus polymyxa with the ability to bind and neutralize endotoxin. Developed in Japan, PMX-DHP is thought to reverse septic vasodilatation by removing circulating endotoxin. A meta-analysis of 28 studies (978 patients)[6] using PMX-DHP in patients with severe sepsis and septic shock demonstrated improved MAP as well as $\mathrm{PaO}_{2} / \mathrm{FiO}_{2}$ ratio in addition to reduced mortality. In 2009, a randomized trial of 64 surgical patients with abdominal septic shock showed improvement of organ function and lower 28-day mortality risk along with improvements in hemodynamic outcome.[7] A more recent meta-analysis of various blood purification trials also revealed decreased mortality in septic patients, and these results were mainly driven by
PMX hemoperfusion.[15] Although blood levels of endotoxin and inflammatory cytokines were not measured in our case, clear hemodynamic improvement after hemoperfusion
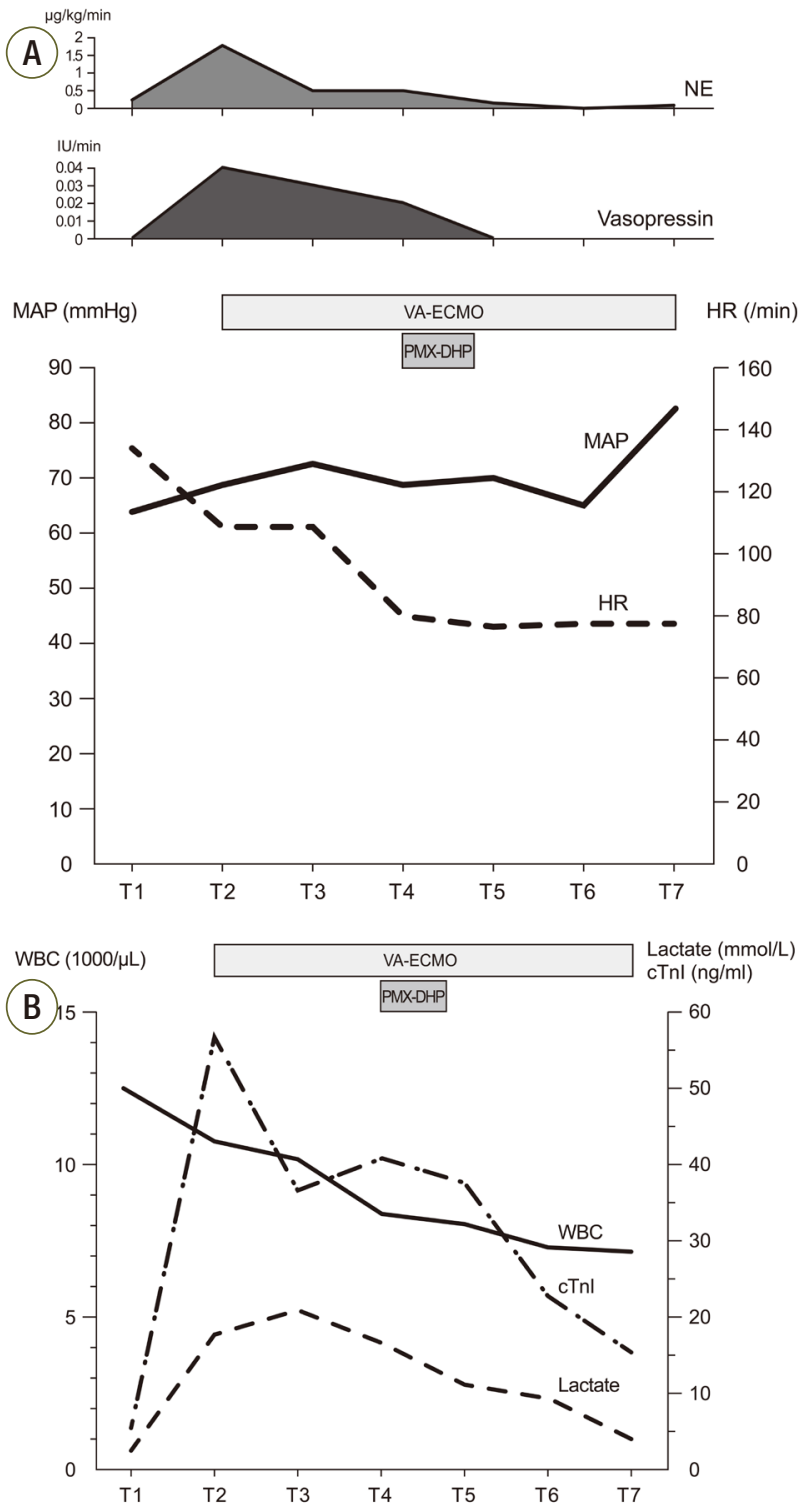

Fig. 4. Graphs show serial changes in vital signs, laboratory findings and vasopressor infusion rate. (A) Trend of MAP, HR and vasopressor infusion rate. (B) Trend of WBC count, lactic acid level and cTnl level. T1: ICU admission, T2: before ECMO support, T3: 1 hour after ECMO support, T4: before PMX-DHP and 14 hours after ECM0, T5: 6 hours after PMX-DHP, T6: 24 hours after PMX-DHP, T7: ECMO removal. MAP: mean arterial pressure; HR: heart rate; WBC: white blood cell; cTnl: cardiac troponin I; ICU: intensive care unit; ECMO: extracorporeal membrane oxygenation, PMX-DHP: polymyxin B directed hemoperfusion; NE: norepinephrine, VA-ECMO: veno-arterial extracorporeal membrane oxygenation. 


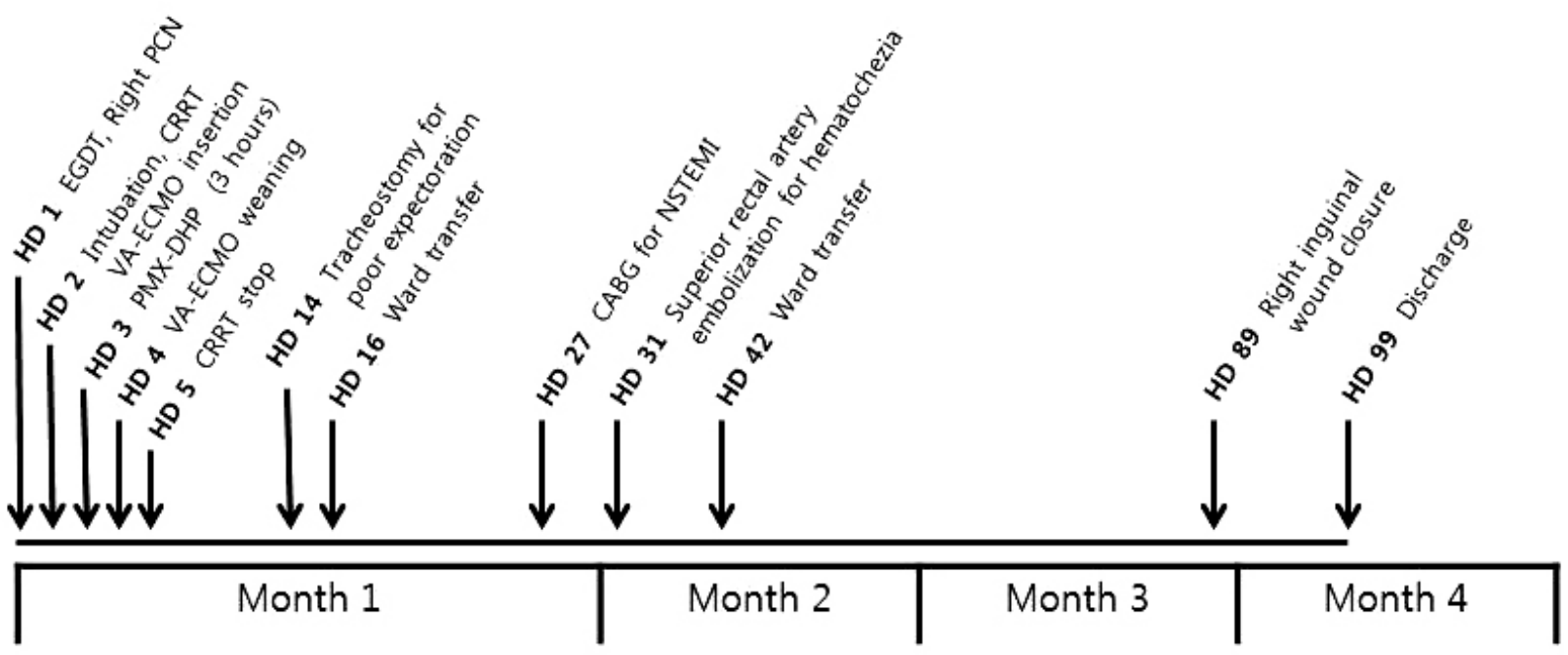

Fig. 5. The brief course of events. EGDT: early goal directed therapy; PCN: percutaneous nephrostomy; CRRT: continuous renal replacement therapy; VA-ECMO: veno-arterial extracorporeal membrane oxygenation; PMX-DHP: polymyxin B directed hemoperfusion; CABG: coronary artery bypass graft surgery; NSTEMI: non-ST segment elevation. myocardial infarction.

was hypothesized to be the outcome of endotoxin removal.

Recently, an animal study showed that the combined use of ECMO and a blood purification device was helpful in the treatment of endotoxemia.[8] In humans, a case of a septic neonate under VA ECMO support treated by PMX-DHP was reported.[10] In our case, septic shock was accompanied by cardiac dysfunction severe enough to require mechanical support by VA ECMO. Endotoxin removal may have played a role in resolving the cardiogenic shock caused by septic cardiomyopathy, since the elevated troponin I level, a biomarker of myocardial damage, was also reduced after hemofiltration.[16] There are two case reports from Japan that suggest PMX-DHP may be beneficial in septic cardiomyopathy by absorbing inflammatory mediators. $[17,18]$

There were no adverse events during the concurrent use of ECMO, CRRT, and hemofiltration in our case. We connected the hemofiltration device parallel to the CRRT device, which were then to the VA-ECMO circuit via three-wayconnectors. Connecting such devices was technically feasible, simple, and safe. This came at no surprise, considering that the arterial flow of ECMO (ranged between $3 \mathrm{~L}$ and $4 \mathrm{~L}$ ) was enough to maintain the slow flow (ranged between 100 $\mathrm{mL}$ and $150 \mathrm{~mL}$ ) required to operate the other devices.

In conclusion, we report the first adult case of the use of PMX-DHP in a patient with septic shock and severe myocardial injury who showed minimal hemodynamic response to VA ECMO support. The positive outcome of using PMXDHP in this patient may be explained by improvement of distributive and cardiogenic shock secondary to the removal of endotoxin. We suggest that PMX-DHP may rescue the refractory septic shock patient with septic cardiomyopathy.

\section{References}

1) Martin GS: Sepsis, severe sepsis and septic shock: changes in incidence, pathogens and outcomes. Expert Rev Anti Infect Ther 2012; 10: 701-6.

2) Bréchot N, Luyt CE, Schmidt M, Leprince P, Trouillet JL, Léger P, et al: Venoarterial extracorporeal membrane oxygenation support for refractory cardiovascular dysfunction during severe bacterial septic shock. Crit Care Med 2013; 41: 1616-26.

3) Park TK, Yang JH, Jeon K, Choi SH, Choi JH, Gwon $\mathrm{HC}$, et al: Extracorporeal membrane oxygenation for refractory septic shock in adults. Eur J Cardiothorac Surg 2015; 47: e68-74.

4) Huang CT, Tsai YJ, Tsai PR, Ko WJ: Extracorporeal membrane oxygenation resuscitation in adult patients with refractory septic shock. J Thorac Cardiovasc Surg 2013; 146: 1041-6.

5) Ronco C, Klein DJ: Polymyxin B hemoperfusion: a 
mechanistic perspective. Crit Care 2014; 18: 309.

6) Cruz DN, Perazella MA, Bellomo R, de Cal M, Polanco N, Corradi V, et al: Effectiveness of polymyxin Bimmobilized fiber column in sepsis: a systematic review. Crit Care 2007; 11: R47.

7) Cruz DN, Antonelli M, Fumagalli R, Foltran F, Brienza N, Donati A, et al: Early use of polymyxin B hemoperfusion in abdominal septic shock: the EUPHAS randomized controlled trial. JAMA 2009; 301: 2445-52.

8) Weerwind PW, van der Veen FH, Gelsomino S, Nagaraj NG, Parise O, Lorusso R, et al: Cytokine removal on extracorporeal life support for treatment of acute endotoxemia: a randomized controlled animal study. Int $\mathrm{J}$ Cardiol 2013; 168: 4699-704.

9) Bruenger F, Kizner L, Weile J, Morshuis M, Gummert JF: First successful combination of ECMO with cytokine removal therapy in cardiogenic septic shock: a case report. Int J Artif Organs 2015; 38: 113-6.

10) Hirakawa E, Ibara S, Tokuhisa T, Hiwatashi S, Hayashida Y, Maede Y, et al: Septic neonate rescued by polymyxin B hemoperfusion. Pediatr Int 2013; 55: e70-2.

11) Court O, Kumar A, Parrillo JE, Kumar A: Clinical review: Myocardial depression in sepsis and septic shock. Crit Care 2002; 6: 500-8.

12) Sharma AS, Weerwind PW, Maessen JG: Extracorpore- al membrane oxygenation resuscitation in adult patients with refractory septic shock. J Thorac Cardiovasc Surg 2014; 147: 1441-2.

13) Akira S: Pathogen recognition by innate immunity and its signaling. Proc Jpn Acad Ser B Phys Biol Sci 2009; 85: $143-56$.

14) Cohen J: The immunopathogenesis of sepsis. Nature 2002; 420: 885-91.

15) Zhou F, Peng Z, Murugan R, Kellum JA: Blood purification and mortality in sepsis: a meta-analysis of randomized trials. Crit Care Med 2013; 41: 2209-20.

16) Nakamura $T$, Ushiyama $C$, Shoji $H$, Koide H: Effects of hemoperfusion on serum cardiac troponin $\mathrm{T}$ concentrations using polymyxin B-immobilized fibers in septic patients undergoing hemodialysis. ASAIO J 2002; 48: 41-4.

17) Nakamura K, Doi K, Inokuchi R, Fukuda T, Hiruma $\mathrm{T}$, Ishii $\mathrm{T}$, et al: Endotoxin adsorption by polymyxin $\mathrm{B}$ column or intraaortic balloon pumping use for severe septic cardiomyopathy. Am J Emerg Med 2013; 31: 893.e1-3.

18) Sugimoto T, Deji N, Isono M, Kume S, Osawa N, Kanasaki K, et al: A case of acute renal failure complicated with Takotsubo cardiomyopathy. Nihon Toseki Igakkai Zasshi 2004; 37: 2093-8. 